* Corresponding author

E-mail address: askrzat@prz.edu.pl

Article information

Article history: AMS-Volume16-No.2-00164-12

Received 15 January 2012

Accepted 31 May 2012

\section{Numerical Analysis of Passive Safety of Street Lighting Posts}

\author{
A. Skrzat \\ Dept. of Materials Forming and Processing, Rzeszow University of Technology, Poland
}

\section{KEY WORDS}

Lighting post, passive safety, dynamic explicit

\begin{abstract}
One of the most important goal in designing of street lighting posts is the fulfillment of requirements of EN 12767 standard which refers to passive safety of road constructions. Lighting posts should reduced overload force when stroked by a passenger car. Experimental investigations involving real posts and cars are relatively expensive, and provide the information about passive safety of existing posts only. In numerical simulations the passive safety of various types of lighting posts might be investigated. Obtained results provide hints about possible modifications necessary to change the level of energy absorption and car passengers safety. The numerical simulation of passenger car - lighting post crash is presented in this paper. The analysis is performed by finite elements method. The dynamic explicit approach is used, which is very effective tool for solving highly non-linear dynamic problems. Original and modified octagonal and conical poles are investigated and the obtained results are compared.
\end{abstract}

\section{Introduction}

In the recent years the fulfillment of EN 12767 requirements which refer to passive safety of road constructions became one of the most important goals in designing of street lighting posts. The European Standard specifies requirements and defines three levels in passive safety of occupants of vehicles impacting with the permanent road structure: energy absorption on high level $(\mathrm{HE})$, energy absorption on low level (LE), and no energy absorption (NE). The manufacturer should select the speed classes under which the supported structure is tested i.e. 35, 70 and $100 \mathrm{~km} / \mathrm{h}$. The level of construction safety [3], [4] depends on two main parameters: acceleration indicator (ASI), and theoretical speed of head impact (THIV). In the impact tests commercially available production passenger car should be used.

Elektromontaz-Rzeszow S.A. from Poland is the manufacturer of modern lighting posts, which satisfy EN 1267 requirements. This company carried out tens of posts crash tests (see Fig. 1).

The experience gained in several crash tests helped engineers from Elektromontaz to introduce some modifications (patented in Polish Patent Office) in order to reach better ASI and THIV category. The increase of passengers safety caused by already made and proposed modifications should be tested in the further experimental investigations. Unfortunately, these tests are relatively expensive and also time consuming. A cheaper and faster manner is the numerical simulation of passenger car - lighting post crash test. The problem is sophisticated from the point of view of mechanics. Large displacements, multi-surface contact, material plastic deformations and inertia loads make this problem highly non-linear. In presented research the problem is solved in fi- 
nite element method. In the numerical simulations the commercial ABAQUS program is used, which is one of the best FEM systems available on the market. The dynamic explicit approach which is very effective in this type of analysis is used.

Numerical simulations of crash test performed for original and modified posts help to select the most promising projects before the real investigations of lighting posts.

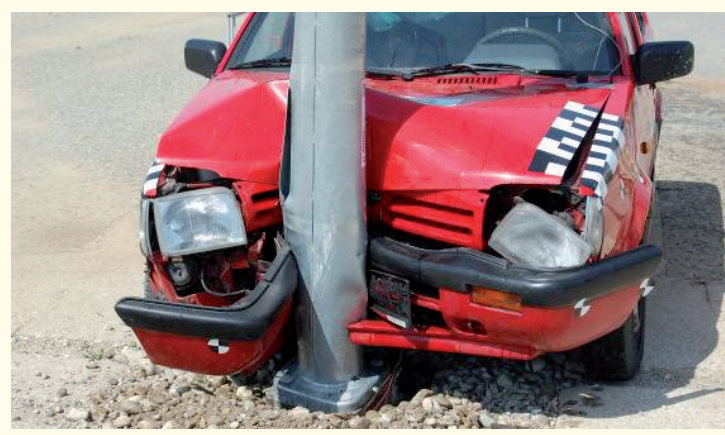

Fig. 1: Lighting post crash test

\section{Dynamic Explicit Method}

The dynamic explicit approach [1] is the method of direct integration of dynamic equations of equilibrium (1)

$$
M^{t} \ddot{u}+C t \dot{u}+K^{t} u={ }^{t} R
$$

where: $M, C$ and $K$ are mass, damping, and stiffness matrices, $\ddot{u}, \dot{u}, u$ is the vector of externally applied loads, are acceleration, velocity, and displacement vectors, respectively. The subscript denotes that this equation is considered for the time $t$.

The differential operators in (1) are replaced by finite difference operators defined in the central difference method as:

$$
\begin{aligned}
& { }^{t} \dot{u}=\frac{-{ }^{t-\Delta t} u+{ }^{t+\Delta t} u}{2 \Delta t} \\
& \ddot{u}=\frac{{ }^{t-\Delta t} u-2{ }^{t} u+{ }^{t+\Delta t} u}{\Delta t^{2}}
\end{aligned}
$$

here is the time increment.

After substituting (3) and (2) into (1) and rearranging one obtains:

$$
\begin{aligned}
& \left(\frac{1}{\Delta t^{2}} M+\frac{1}{2 \Delta t} C\right) t+\Delta t \\
& u={ }^{t} R-\left(K-\frac{2}{\Delta t^{2}} M\right){ }^{t} u \\
& -\left(\frac{1}{\Delta t^{2}} M-\frac{1}{2 \Delta t} C\right){ }^{t-\Delta t} u
\end{aligned}
$$

Equation (4) allows to find the solution ${ }^{t}+\Delta t u$ if solutions ${ }^{t-\Delta t} u$ and ${ }^{t} u$ are known. A special starting procedure is needed for the time $t=0$. The initial boundary conditions $\left({ }^{0} \ddot{u},{ }^{0} \dot{u},{ }^{0} u\right.$ are known) are used to provide $-\Delta t u$.

$$
\begin{aligned}
& { }^{0} \dot{u}=\frac{-{ }^{-\Delta t} u+{ }^{\Delta t} u}{2 \Delta t} \\
& { }^{0} \ddot{u}=\frac{{ }^{-\Delta t} u-2^{0} u+{ }^{\Delta t} u}{\Delta t^{2}}
\end{aligned}
$$

Thus:

$$
{ }^{-\Delta t} u={ }^{0} u-\Delta t^{0} u+\frac{\Delta t^{2}}{2} \ddot{u}
$$

The dynamic explicit approach is conditionally stable i.e. $\Delta t$ should be small enough [2]. The stable time increment depends e.g. on the size of the smallest finite element. For sophisticated geometry the finite element mesh should be generated very carefully to avoid the existence of very small elements, therefore. If such elements are generated the stable time increment is less than $10^{-6} \mathrm{sec}$ and the solution is time-consuming.

The integration of (1) becomes very effective if velocity-dependent damping is neglected, and mass matrix is diagonal (lumped mass matrix). In such case no global stiffness matrix of the complete element assemblage needs to be calculated, and very large problems are being solved effectively.

\section{FEM Vehicle Model}

The proper response of the lighting post to sudden crash caused by the passenger car requires correct modeling of both structures [5]. The front of the real car consists of many deformable elements. Taking all of them into consideration would caused very complicated numerical model. The solution would require use of the powerful workstation, and will be very time-consuming. The car model used in presented simulations was as simple as possible (Fig. 2). It consists of the following parts: car body, wheels, engine and the front bumper. The engine is modeled by solid elements. Shell elements are used to model all other car parts. The total mass and the mass center are as those required by EN 12767 i.e. $900 \mathrm{~kg} \pm 40 \mathrm{~kg}$, centre of gravity height in distance from ground $0,49 \mathrm{~m} \quad \pm 0,7 \mathrm{~m}$. 


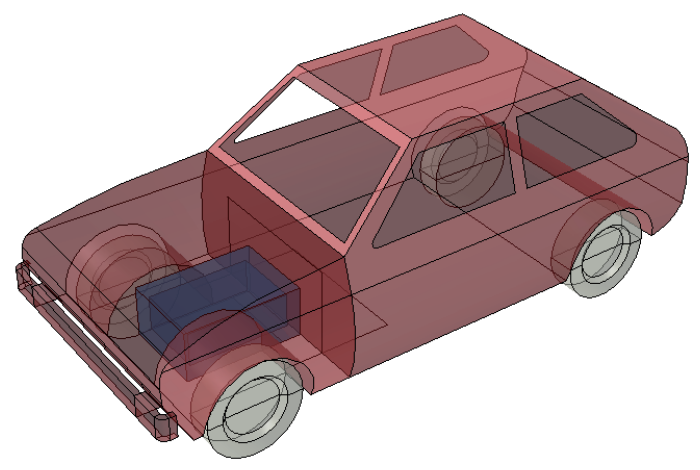

Fig. 2: The geometry of simplified car model.

The front characteristics of the vehicle model is selected in the solution of calibration test. In the calibration test the vehicle should impact a vertical rigid cylinder of diameter $290 \mathrm{~mm} \pm 20 \mathrm{~mm}$ at 35 $\mathrm{km} / \mathrm{h}$. According to EN 12767 the car should stop in 80-100 ms. The geometric model of calibration test is shown in Fig. 3, while the computed plot of velocity versus time obtained for the assumed car model is presented in Fig.4.

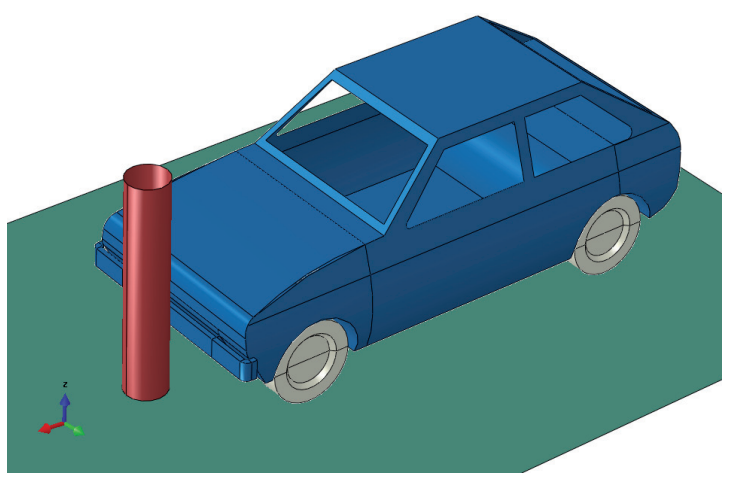

Fig. 3: The calibration test.

The velocity is measured in the car mass center. Fig. 4. shows that the car model satisfies the requirements of EN 12767 considering the calibration test.

\section{FEM Simulation of the Crash Test}

Two magnitudes of car impact velocity are considered: 35 and $100 \mathrm{~km} / \mathrm{h}$. Two basic types of lighting posts are investigated: conical and octagonal. Car and post models are assembled over the ground level. Elastic-plastic properties of post and car material are considered. The strain hardening characteristics of the post material is found in the experimental investigations. The Hayer method in uniaxial tension test is used to obtain material plastic coefficients.

The so-called ABAQUS general contact is defined. The general contact assumes possible contact between all surfaces in the model including selfcontact. The foundation of the lighting post is fixed. The initial velocity is applied to the car model. The gravity forces are applied the car model to avoid jumps after crashing the post. The dynamic explicit approach is chosen as the solution procedure. Large displacements and strains are considered.

The enlargement of finite element mesh in the neighborhood of the car front is presented in Fig. 5. Because the lighting post is the main object of interest the finite element mesh generated for the post is very dense in order to obtain the results of acceptable precision. Regular finite element mesh is generated wherever possible.

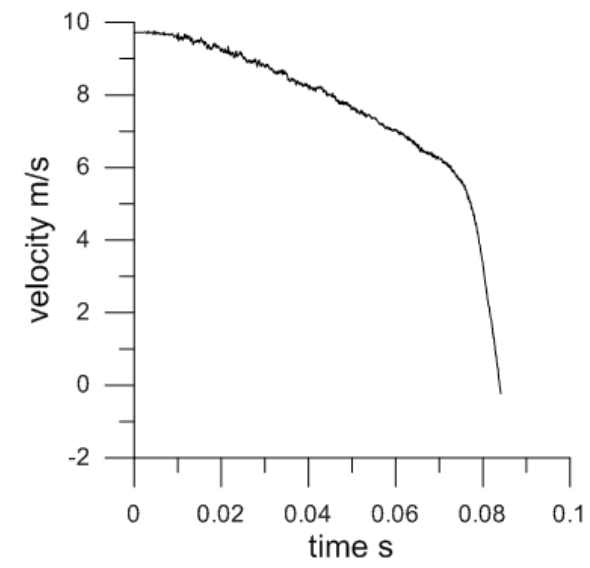

Fig. 4: Velocity vs. time in calibration test.

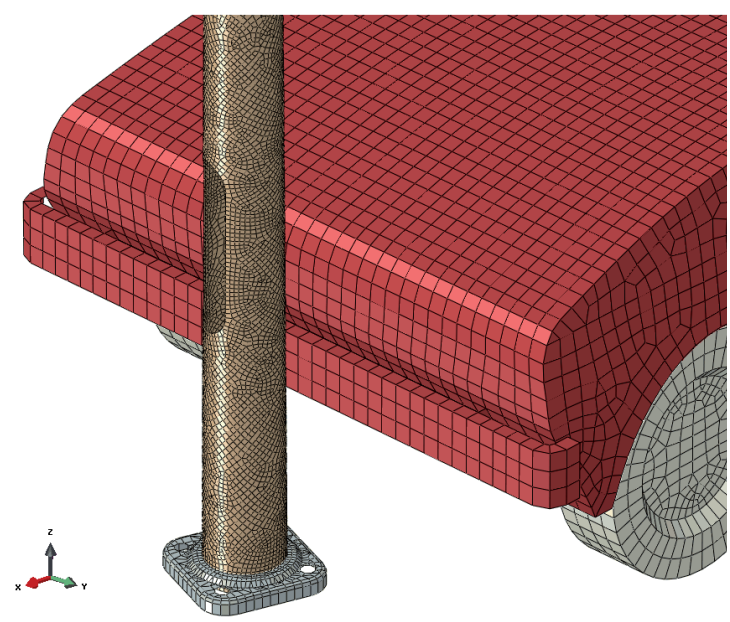

Fig. 5: The finite element mesh of lighting post and car. 
The generation of finite element mesh should be made very carefully. Some small surfaces e.g. welding slot should be combined with larger ones. This way generation of very small finite elements is avoided and the stable solution increment becomes larger. Some parts should be coupled (connected) e.g. the post and the post foundation. Car wheels are constrained to the car body (in this simplified car model the suspension system is not considered).

Some model and the solution data of the crash test analysis are as follows:

\section{- number of degrees of freedom: 194000, \\ - stable time increment: $2.3 e^{-7} \mathrm{~s}$, \\ number of increments: 125000 , \\ - computation time on PC: 16 hours.}

Many finite element method simulations of the crash test are made in this research. In Fig. 6 presented is an exemplary solution. The initial car velocity is $35 \mathrm{~km} / \mathrm{h}$, the considered analysis time $\mathrm{t}=0.06 \mathrm{~s}$. The von Mises stress contours are shown on deformed shape. The largest stresses are located in the lamp foundation and in the neighborhood of maintenance opening. Maximum stress magnitude exceeds the yielding stress. Although the stress distribution provides information about yielding occurrence interesting for designers, in this simulations the deformed shape is the main quantity of interest.

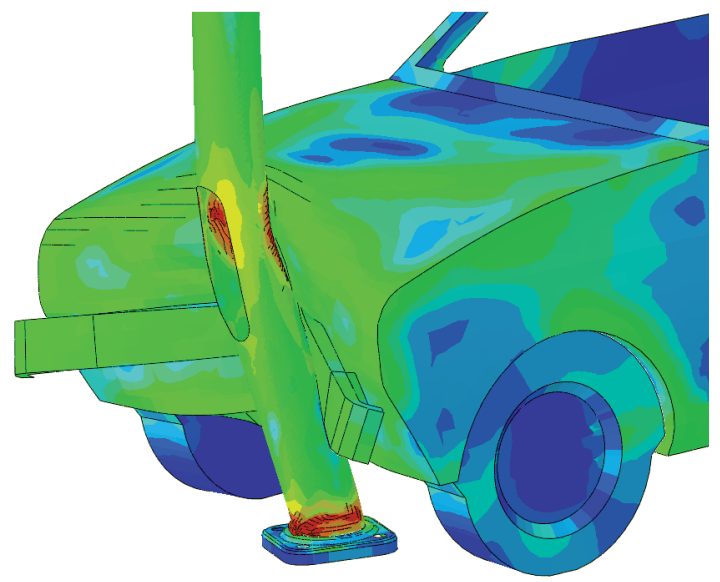

Fig. 6: Numerical simulation of the crash test, initial speed $35 \mathrm{~km} / \mathrm{h}$.

The deformations shown in Fig. 6 are comparable to the post and car deformations appearing in real crash test (Fig. 7). It proves that mathematical and numerical models assumed in FEM simulations of the crash test are correct. Comparison of deformations of original and modified lighting post should provide valuable information about the influence of proposed modifications on safety parameters. From the point of view of passengers safety the maximum acceleration appearing in the crash test is a very important factor. In EN 12767 standard the level of safety represented by acceleration indicator ASI and theoretical speed of head impact THIV, depends on acceleration magnitude occurred in the crash test.

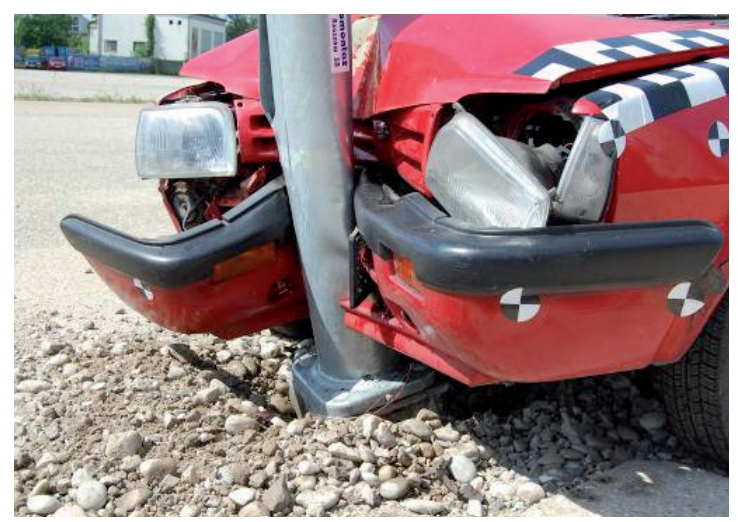

Fig. 7: Deformations in real crash test.

In numerical simulations presented in this paper the acceleration of the car is registered in the car mass center. The variation in time of computed car acceleration for initial velocity $35 \mathrm{~km} / \mathrm{h}$ is shown in Fig. 8.

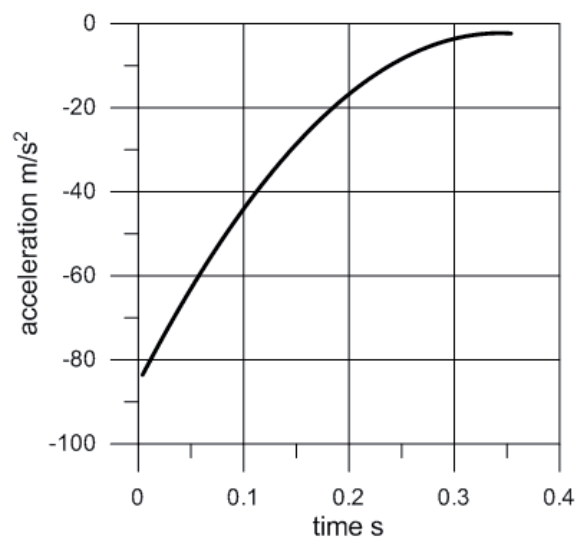

Fig. 8: Vehicle acceleration vs. time in the crash simulation, initial speed $35 \mathrm{~km} / \mathrm{h}$. 
In this particular test the absolute maximum acceleration is about $8 \mathrm{~g}$. Similar plots to Fig. 8 obtained for simulations performed for modified lighting posts provides information about the influence of proposed modifications on the safety level.

Several numerical simulations are made for initial vehicle speed $100 \mathrm{~km} / \mathrm{h}$. Experimental investigations have shown, that for this velocity the foundation of the lighting tends to bent or break. The car goes over the post which is put on the ground and braking is not very rapid, therefore. If the post is put on the ground the requirements of EN 12767 standard are satisfied without difficulties. In this case the standard rules may be only violated by the strike of the lighting post into the car roof or into the car front window (see Fig. 9). Properly designed road constructions for the high-speed impact should compromise tension and bending stiffness, therefore.

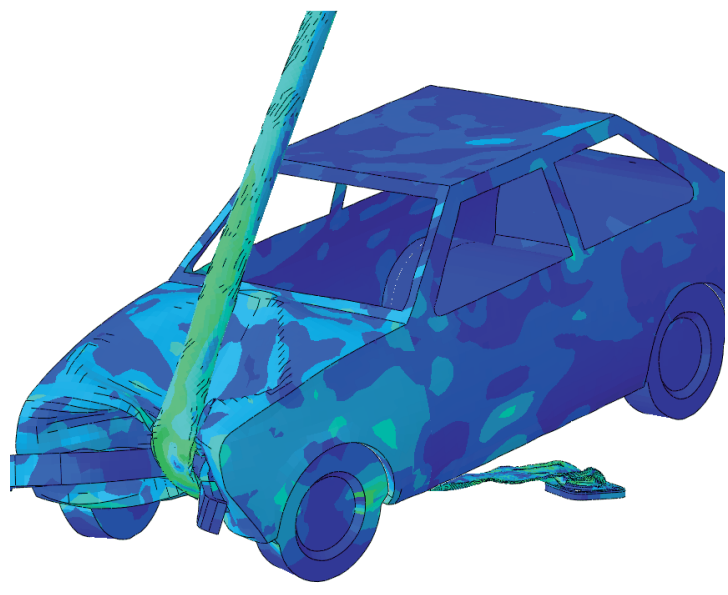

Fig. 9: Numerical simulation of the crash test, speed $100 \mathrm{~km} / \mathrm{h}$.

Fig. 10 shows the deformations of the lighting post hit by the car going with the initial speed 100 $\mathrm{km} / \mathrm{h}$. In this simulation no serious damage of the post foundation is considered.

The plot of vehicle acceleration center versus time computed in numerical simulation at the car mass is shown in Fig. 11. This characteristic is different to one presented in Fig. 8. It is caused by the large post bending. as a result of this bending the post is put on the ground.

In this simulation the bent lighting post has slightly hit the right side of the car roof.

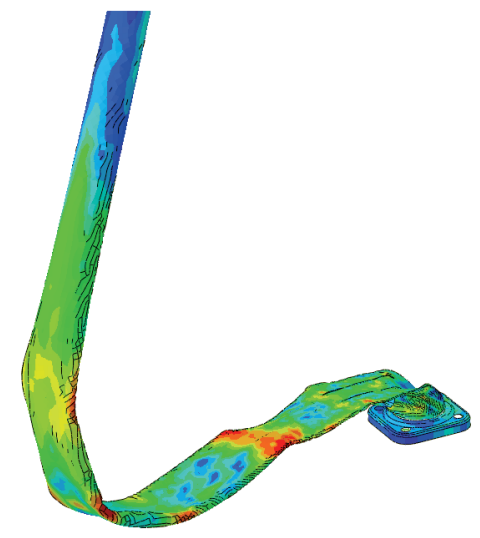

Fig. 10: Lighting post deformations, initial speed $100 \mathrm{~km} / \mathrm{h}$.

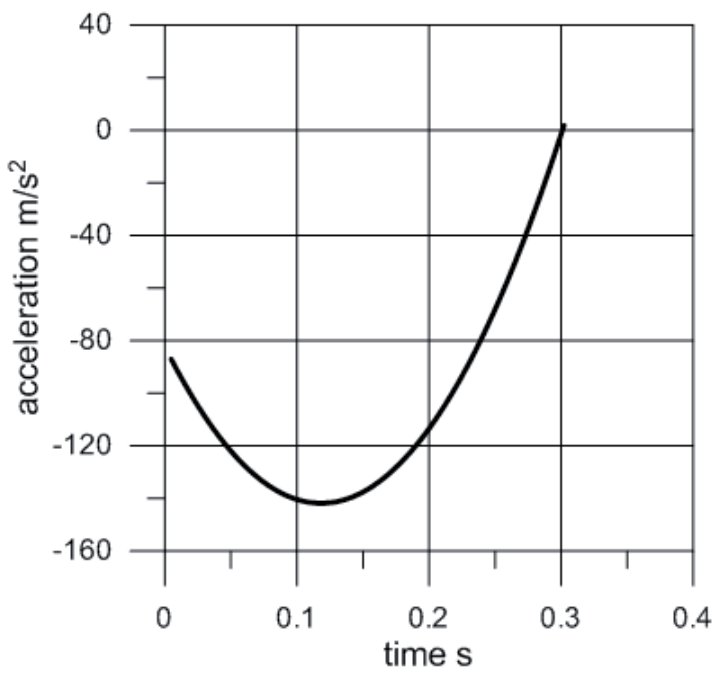

Fig. 11: Vehicle acceleration vs. time in the crash simulation, initial speed $100 \mathrm{~km} / \mathrm{h}$.

\section{Modifications of Lighting Posts}

The main advantage of numerical simulations of the crash test over real experiments is the possibility of investigation of influence of construction modifications on the safety indicators ASI and THIV. Numerical simulations do not require existence of the new lighting post prototypes. The cost of numerical computations is very low when compared to the cost of real experiments. Simulations made for several modified posts help to select the most promising constructions. Moreover, the results of numerical tests contain detailed information about deformations, strains, stresses, variation of velocity, acceleration, and energy, which is hardly available or not available in real experiments even for use of 
advanced measurement equipment. If the real test is successfully simulated on computer, the results of numerical simulations extend those obtained in experimental investigations. In engineering practice this results give a better insight into the crash mechanics.

Of course, in order to receive ASI and THIV certificate of conformity real investigations have to be made. When the crash test is simulated on computer and the preliminary numerical results are already known, the real tests have to be performed only for a few (typical or modified) posts.

Engineers from Elektromontaz S.A. proposed some modification of lighting posts in order to increase the posts passive safety. Designing details are the secret of manufacturer and cannot be described here. Generally, these modifications consider changes of thickness and additional cuts made in several locations in posts.

A number of numerical simulations of the crash test is made for modified posts. Results of this analysis are promising. In Fig. 12 presented are plots of the car acceleration during impact in typical and modified lighting post. The initial car velocity is 35 $\mathrm{km} / \mathrm{h}$.

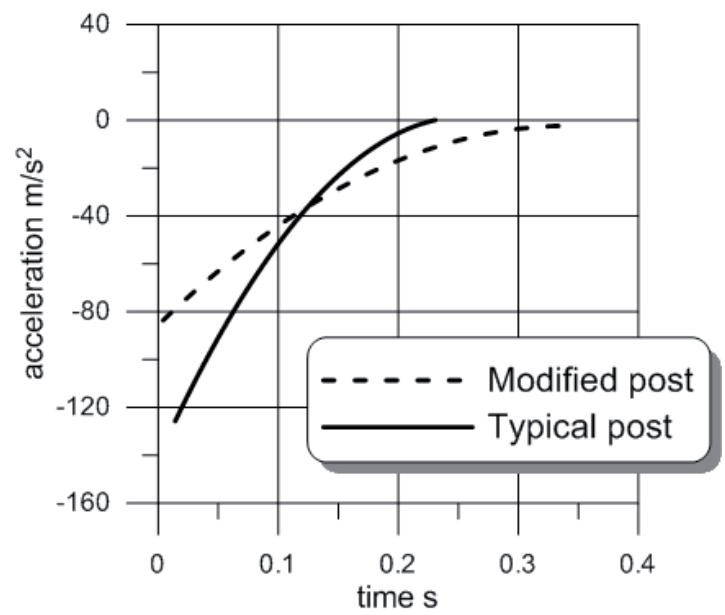

Fig. 12: Vehicle acceleration vs. time in the crash simulation, initial speed $35 \mathrm{~km} / \mathrm{h}$.

One can clearly seen that for the modified lighting post the car braking time is extended, and the maximum car acceleration is significantly decreased. The proposed lighting post modifications have positively changed the passive safety conditions.

\section{Conclusion}

In the recent years the progress in computer software and hardware allowed engineers to simulate reality without making prototypes. Numerical simulations allow for analyzing of many construction parameters. The most promising concepts may be chosen before the prototype is made. The crash test simulation is a very good example of modern engineering practice. The car passengers passive safety became one of the most important challenge of road constructions manufacturers. Finite element method simulations of standard crash test help designers to develop better constructions. Polish manufacturer of lighting posts Elektromontaz Rzeszow S.A. carried out tens of posts crash tests. Recently, advanced commercial software is used in order to numerically simulate the crash test. Numerical simulations of crash tests presented in this research provide the results comparable with measurements made in real investigations. It proves the correctness of proposed numerical model. FEM solutions are obtained for the modified lighting posts. The analysis results confirm the increase of passive safety parameters in modified posts. The cost of numerical computations is very low when compared to the cost of real experiments. Detailed information about deformations, strains and stresses resulting from numerical tests help engineers for better understanding the crash mechanics and make up correct decisions in the new projects.

\section{References}

[1] Bathe K.J., Finite Element Procedures, Prentice Hall, Upper Saddle River, 1996

[2] Belytschko T., Liu W.K., Moran B., Nonlinear Finite Elements for Continua and Structures, Wiley, West Sussex, 2000.

[3] Harless D., Hoffer G., Do laboratory frontal crash test programs predict driver fatality risk?, Accident Analysis and Prevention, vol 39, 2007, p. 902-913.

[4] Jermakian J., Crash avoidance potential of four passenger vehicle technologies, Accident Analysis and Prevention, vol 43, 2011, p. 732-740

[5] Ren Z., Vesenjak M., Computational and experimental crash analysis of the road safety barrier, Engineering Failure Analysis, vol. 12, 2005, p. 963-973. 